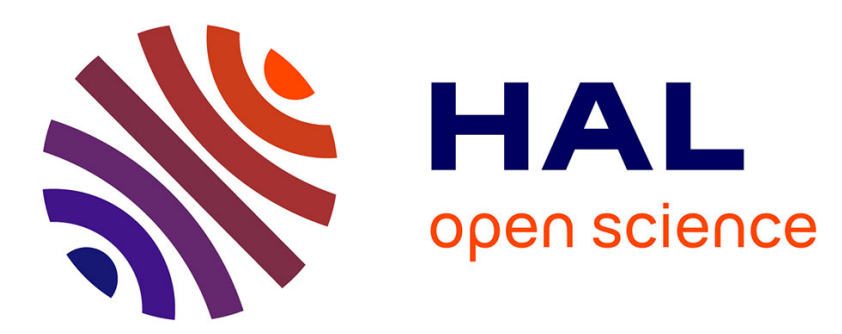

\title{
Effect of Preservation Period on the Viscoelastic Material Properties of Soft Tissues With Implications for Liver Transplantation
}

\author{
Sina Ocal, M. Umut Ozcan, Ipek Basdogan, Cagatay Basdogan
}

\section{To cite this version:}

Sina Ocal, M. Umut Ozcan, Ipek Basdogan, Cagatay Basdogan. Effect of Preservation Period on the Viscoelastic Material Properties of Soft Tissues With Implications for Liver Transplantation. Journal of Biomechanical Engineering, 2010, 132 (10), pp.101007. 10.1115/1.4002489 . hal-03177341

\author{
HAL Id: hal-03177341 \\ https://hal.science/hal-03177341
}

Submitted on 23 Mar 2021

HAL is a multi-disciplinary open access archive for the deposit and dissemination of scientific research documents, whether they are published or not. The documents may come from teaching and research institutions in France or abroad, or from public or private research centers.
L'archive ouverte pluridisciplinaire HAL, est destinée au dépôt et à la diffusion de documents scientifiques de niveau recherche, publiés ou non, émanant des établissements d'enseignement et de recherche français ou étrangers, des laboratoires publics ou privés. 


\title{
Effect of Preservation Period on the
}

\section{Viscoelastic Material Properties of Soft Tissues}

\section{with Implications for Liver Transplantation}

\author{
Sina Ocal, M. Umut Ozcan, Ipek Basdogan, Cagatay Basdogan* \\ College of Engineering, Koc University, Istanbul, 34450, Turkey \\ \{sinocal,uozcan,ibasdogan,cbasdogan\}@ku.edu.tr
}

\begin{abstract}
The liver harvested from a donor must be preserved and transported to a suitable recipient immediately for a successful liver transplantation. In this process, the preservation period is the most critical, since it is the longest and most tissue damage occurs during this period due to reduced blood supply to the harvested liver and change in its temperature. We investigate the effect of preservation period on the dynamic material properties of bovine liver using a viscoelastic model derived from both impact and ramp and hold experiments. First, we measure the storage and loss moduli of bovine liver as a function of excitation frequency using an impact hammer. Second, its time-dependent relaxation modulus is measured separately through ramp and hold experiments performed by a compression device. Third, a Maxwell solid model that successfully imitates the frequency- and time-dependent dynamic responses of bovine liver is developed to estimate the viscoelastic material coefficients by minimizing the error between the experimental data and the corresponding values generated by the model. Finally, the variation in the viscoelastic material coefficients of bovine liver are investigated as a function of preservation period for the liver samples tested 1, 2, 4, 8, 12, 24,
\end{abstract}

\footnotetext{
* Corresponding author:

College of Engineering

Koc University

Istanbul, 34450, Turkey

Tel: $90+212-338-1721$

Fax: 90+ 212-338-1548

e-mail: cbasdogan@ku.edu.tr

C. Basdogan
}

BIO-10-1131 
36, and $48 \mathrm{hrs}$ after harvesting. The results of our experiments performed with three animals show that the liver tissue becomes stiffer and more viscous as it spends more time in the preservation cycle.

\section{Introduction}

Today, treatment of severe liver failure is not possible unless the diseased organ harvested from the body and replaced with a healthy liver, which is known as liver transplantation. Unfortunately, the number of liver donors is significantly smaller than the patients who need healthy organs. The sources of liver donors are tried to be increased by using living and deceased donors and the techniques of split and domino transplants. While the success rate with deceased donors is low, they still hold a considerable part in liver transplantation donor sources. Typically, the donor and the recipient are in different locations, which bring up the problem of the preservation. The liver harvested from a donor must be preserved and transported ex vivo with effective, safe and reliable methods and after that transplanted to a suitable recipient immediately. Along this process, tissue damage occurs in the liver due to drop in its temperature (hypothermia) and insufficient supply of blood to its vessels (ischemia). While the effect of preservation period on the cell structure of animal and human livers have been investigated extensively, the same effect on the gross material properties of liver tissue has not been studied before.

Most of the earlier research studies conducted with animal and human livers have focused on the investigation of static material properties (Carter et al., 2001, Ottensmeyer, 2001, Tay et al., 2006, Samur et al., 2007, Roan and Vemaganti, 2007, Nava et al., 2008). The number of studies investigating the dynamic material properties of animal and human livers are much 
less than the ones investigating the static material properties. In most of these studies, either time- or frequency-dependent material properties have been measured via stress relaxation and dynamic loading experiments, respectively. Liu and Bilston (2000) investigated the linear viscoelastic properties of bovine liver via three different experiments a) shear strain sweep oscillation, b) shear stress relaxation, and c) shear oscillation. The results of relaxation experiments show that the shear modulus reaches to steady state around $0.6 \mathrm{kPa}$. The results of the oscillatory shear experiments show that the storage modulus increases from $1 \mathrm{kPa}$ to 6 $\mathrm{kPa}$ with increasing frequency and the loss modulus is less than $1 \mathrm{kPa}$, increases to a peak at about $1 \mathrm{~Hz}$ and then decreases to $0.4 \mathrm{kPa}$ as the frequency reaches to $20 \mathrm{~Hz}$. Kruse et al. (2000) utilized magnetic resonance elastography (MRE) and estimated the average shear modulus of porcine liver as $2.7 \mathrm{kPa}$ for 5 different animals at 6 different wave frequencies ranging from 75-300 Hz. Kiss et al. (2004) performed in vitro experiments with canine liver tissue to characterize its dynamic response by applying cyclic stimuli to the tissue. They calculated the storage and the loss moduli of the liver tissue from the frequency-dependent complex elastic modulus as $1-10 \mathrm{kPa}$ for the frequencies ranging from 0.1 to $400 \mathrm{~Hz}$. Valtorta and Mazza (2005) developed a torsional resonator to characterize the dynamic material properties of bovine and porcine liver for the frequency range of 1-10 kHz. The results of the in-vitro experiments on porcine liver show that the magnitude of complex shear modulus varies between 5-50 kPa depending on whether the data collected from the external surface or the internal section of the liver. The shear modulus of bovine liver was found to vary between 15-30 kPa. Zhang et al. (2007) characterized the frequency-dependent viscoelastic properties of fresh veal liver using two independent methods: crawling wave estimator (CRE) and the mechanical measurement (MM). In CRE method, the liver samples were placed between piezoelectric shear wave sources and the resulting crawling wave movies were captured using ultrasound scanners to estimate the elastic modulus in frequency range from 80 to $280 \mathrm{~Hz}$. In 
MM method, stress relaxation experiments were performed with a mechanical compression device and the complex elastic modulus was obtained from time domain response via Fourier transform fro the same frequency range. The results of the experiments showed that the magnitude of the complex elastic modulus of veal liver varied from 10-40 kPa and increased with frequency in the tested range. Saraf et al. (2007) investigated the dynamic response of human liver in hydrostatic compression and simple shear using the Kolsky bar technique at high strain rates ranging from 300 to $5000 \mathrm{~s}-1$. They measured the bulk and the shear moduli of human liver under dynamic loading as $280 \mathrm{kPa}$ and $37-340 \mathrm{kPa}$ (depending on the strain rate), respectively.

In this article, we investigate the effect of preservation period on the dynamic (both time and frequency-dependent) material properties of bovine liver. For this purpose, we first measure the frequency-dependent force response of bovine liver samples using an impact hammer for different preservation periods up to 48 hours. To our knowledge, this is the first time that the frequency-dependent properties of a liver tissue are characterized by using an impact hammer. Second, we measure the time-dependent relaxation response of the same liver samples by conducting ramp and hold experiments via a separate compression device. Third, we fit the data collected from the both experiments (relaxation and impact) to a Generalized Maxwell Solid (GMS) model to obtain the optimum viscoelastic material coefficients. The previous investigators modeling the dynamic response of soft tissues have typically relied on the experimental data collected from one type of experiment only. Either relaxation or dynamic (cyclic) loading experiments are performed to model time- or frequency-dependent material properties of the soft tissues being tested, respectively. As a final step, we investigate the effect of preservation period on the response of this model. To our knowledge, there is no 
earlier study in the literature investigating the effect of preservation period extensively on the gross mechanical properties of animal or human liver.

\section{Materials and Methods}

\subsection{Sample Preparation}

The livers harvested from 3 different animals were used in the experiments. After harvesting, the livers were flushed and preserved with Lactated Ringer's (LR) solution at $4{ }^{0} \mathrm{C}$. During the preservation period, each liver was kept in a commercial cooler and the temperature was controlled by a digital thermometer. Cylindrical samples were obtained from each liver at different time steps: $1,2,4,8,12,24,36$, and $48 \mathrm{hrs}$ after harvesting. All the samples were taken from the right lobe of livers for consistency. The diameter and the length of the samples were $50 \mathrm{~mm}$ and $25 \mathrm{~mm}$, respectively. We selected the sample sizes such that they can preserve their shape after they are harvested from the livers and do not buckle during the experiments. Before the experiments, the samples were covered by Vaseline to prevent fluid loss and dehydration. Since less damage is made to the sample in impact experiments, first the impact and then the ramp and hold tests were performed on each sample.

\subsection{Impact Experiments}

The response of a test specimen under impact loading can be modeled using a hysteretic damping model as shown below (Nashif, 1985):

$m \ddot{x}(t)+k^{*} x(t)=f(t)$ 
where $m$ is the mass of the pre-load placed on the specimen, $k^{*}$ is the complex stiffness of the specimen, $f(t)$ is the excitation force, which results in a displacement $x(t)$. The same equation can be written in the frequency domain to obtain the following transfer function (also known as the frequency response function, FRF)

$T(j \omega)=\frac{X(j \omega)}{F(j \omega)}=\frac{1}{-m \omega^{2}+k(\omega)(1+j \eta(\omega))}$

where, $k(\omega)$ is the dynamic stiffness and $\eta(\omega)$ is defined as the loss factor. Now, if we define $\mathrm{r}$ as the ratio of the excitation frequency to the natural frequency, $r=\omega / \omega_{n}$, then the complex stiffness and the loss factor of the specimen can be calculated from the measured transfer function and the resonance frequency as suggested in Lin et al. (2005)

$k(\omega)=\frac{\operatorname{Re}(T(j \omega))}{|T(j \omega)|\left(1-r^{2}\right)}$

$\eta(\omega)=-\frac{\operatorname{Im}(T(j \omega))}{\operatorname{Re}(T(j \omega))}\left(1-r^{2}\right)$

After obtaining the dynamic stiffness, the dynamic elastic modulus, $E(\omega)$, can be calculated using the following relation

$E(\omega)=\frac{k(\omega) L}{A}$ 
where, $L$ is the length of the specimen in the direction of the loading and $A$ is the cross sectional area of the sample. Now, similar to the complex stiffness term appearing in Eq. 1, the complex elastic modulus can be written as

$E^{*}(\omega)=E(\omega)(1+\eta(\omega) j)$

Alternatively, it can be written in terms of real and imaginary parts as

$E^{*}(\omega)=E_{S}(\omega)+j E_{L}(\omega)$

The real part, $E_{S}(\omega)$, is known as the storage modulus and it is an indicator of energy storage capacity of the viscoelastic material. The imaginary part, $E_{L}(\omega)$, is known as the loss modulus and it is related the energy dissipation capacity of the material.

In our experiments, an impulse excitation force was applied to a pre-load mass (400 gram) placed on top of each liver sample using an impact hammer (PCB Piezotronics Inc., Model 086C03, sensitivity is $2.1 \mathrm{mV} / \mathrm{N}$ ) equipped with a force sensor (Figure 1). Note that the weights of the all liver samples ( $40 \pm 3$ grams) were significantly smaller than the weight of the preload. The cross-sectional area of the preload was equal to the cross-sectional area of the samples. For better response at low frequencies, a soft tip and an extender mass were utilized as suggested by the manufacturer. The impulse response of the specimen was measured by a piezoelectric accelerometer (PCB Piezotronics Inc., Model 333B30, sensitivity is $101.2 \mathrm{mV} / \mathrm{g}$, where $\mathrm{g}$ is the gravitational acceleration; range is $0.5-3000 \mathrm{~Hz}$ ). The accelerometer was attached to the pre-load mass using a thin film of adhesive wax. As suggested by the manufacturer, five measurements were taken from each specimen and the 
average values were used in the analysis. The accelerometer and the force sensor were connected to a dynamic signal analyzer (Data Physics Corporation, type SignalCalc Mobilyzer) to record the data and calculate the frequency response function (FRF). The frequency response function (FRF) was obtained by taking the Fast Fourier transform of the impulse response. Then, the storage and loss moduli of the liver samples for different preservation periods were calculated as a function of frequency using the Eqs (1-6).

$* * * * * * * * * * * * * * * *$ Insert Figure $1 * * * * * * * * * * * * * * * *$

\subsection{Ramp and Hold Experiments}

In ramp and hold experiments, stress relaxation responses of the same liver samples were measured for different preservation periods to estimate the time-dependent relaxation modulus, $\mathrm{E}_{\mathrm{R}}(t)$. For this purpose, a separate experimental set-up was developed to apply compressive strains to the liver samples and measure their force response through a force sensor (Figure 2). The major components of this set-up include a high-torque step motor moving a compression plate on a power screw and a force sensor attached to the shaft of the compression plate. The step motor (Intelligent Motion Systems Inc., model MDrive23Plus, 51200 steps/rev) was programmed to compress the liver samples in vertical direction at a user-specified rate using the compression plate. As the sample was compressed, the force response was measured using a force transducer (ATI Industrial Automation Inc., model Nano 17) having a force range of $17 \mathrm{~N}$ in the normal direction, $12 \mathrm{~N}$ in other principal directions and a resolution of $1 / 160 \mathrm{~N}$ along each of the three orthogonal axes. The force data was acquired using a 16-bit analog input card NI PCI-6034E (National Instruments Inc.) with a maximum sampling rate of $200 \mathrm{kS} / \mathrm{s}$. 
$* * * * * * * * * * * * * * * *$ Insert Figure $2 * * * * * * * * * * * * * * * *$

In our experiments, the liver specimens were compressed to $4.8 \mathrm{~mm}$ in $0.1 \mathrm{~s}$ and the compression plate was held there for 500 seconds to record the force relaxation response as a function of time. A total of 9 measurements were made at 1, 2, 4, 8, 12, 18, 24, 36 and 48 hours after harvesting. To obtain the stress relaxation modulus, $E_{R}(t)$, the recorded force values were divided by the cross sectional area of the samples and the strain.

\subsection{Viscoelastic Tissue Model}

The time-dependent viscoelastic material properties of soft tissues are typically characterized by ramp and hold experiments in biomechanics literature. When a soft organ tissue is subjected to a ramp and hold strain, the stress response at that strain decreases exponentially with time, reaching to a steady state value. This is explained by the phenomena of stress relaxation under constant strain and can be characterized by a time-dependent relaxation modulus, $\mathrm{E}_{\mathrm{R}}(\mathrm{t})$. If a GMS is used for modeling the viscoelastic behavior of a soft tissue (Figure 3), then the time-dependent relaxation modulus of the tissue can be obtained analytically from its stress response to a constant strain input as

$$
E_{R}(t)=E_{0}\left[1-\sum_{j=1}^{N} \alpha_{j}\right]+E_{0} \sum_{j=1}^{N} \alpha_{j} e^{-t / \tau_{j}}
$$

This representation is also known as the Prony series. The response of the same viscoelastic model to an impact loading (or equivalently cyclic loading) enables us to calculate the storage and loss moduli as a function of excitation frequency as 
$E_{S}(\omega)=E_{0}\left[1-\sum_{j=1}^{N} \alpha_{j}\right]+E_{0} \sum_{j=1}^{N} \frac{\alpha_{j} \tau_{j}{ }^{2} \omega^{2}}{\left(1+\tau_{j}{ }^{2} \omega^{2}\right)}$

$E_{L}(\omega)=E_{0} \sum_{j=1}^{N} \frac{\alpha_{j} \tau_{j} \omega}{\left(1+\tau_{j}^{2} \omega^{2}\right)}$

In above equations $(7,8,9), E_{0}$ is the short-term elastic modulus, $\alpha_{j}=E_{j} / E_{0}$ is the relative modulus and $\tau_{j}=b_{j} / E_{j}$ is the time constant, where $b_{j}$ represents the damping coefficient and $\mathrm{N}$ is the number of terms (i.e. Maxwell arms) used in the GMS model. Note that the long term modulus, which determines the steady state response, is related to the short term modulus through the relative moduli, $E_{\infty}=E_{0}\left(1-\sum_{j=1}^{N} \alpha_{j}\right)$.

$* * * * * * * * * * * * * * * *$ Insert Figure $3 * * * * * * * * * * * * * * * *$

In our approach, the GMS model integrates the experimental data acquired by the relaxation and impact tests via optimization (Figure 3). The goal of the optimization is to estimate the number of Maxwell arms (N) and the material coefficients $E_{0}, \alpha_{\mathrm{j}}$, and $\tau_{j}$ in the GMS model by minimizing the error between the experimental data and the corresponding values generated by the GMS model. Hence, the error function to be minimized, $F_{\min }$, can be defined as

$$
F_{\min }=\sum_{i=1}^{M}\left\{\left[E_{R}^{\exp }(t)-E_{R}^{\bmod }(t)\right]^{2}+\left[E_{S}^{\exp }(\omega)-E_{S}^{\bmod }(\omega)\right]^{2}+\left[E_{L}^{\exp }(\omega)-E_{L}^{\bmod }(\omega)\right]^{2}\right\}
$$


where, $E^{\exp }$ and $E^{\text {mod }}$ represent the moduli obtained from the experimental data and calculated from the model, respectively, and $\mathrm{M}$ is the number of data points used for the optimization.

\section{Results and Discussion}

Figure 4 presents the experimental data of the impact test for one animal. Note that due to the singularities at $r=1$ in Eq. 3, large variations occur around the resonance frequency. As shown, the storage and loss moduli of bovine liver increase with an increase in preservation period.

$* * * * * * * * * * * * * * * *$ Insert Figure $4 * * * * * * * * * * * * * * * *$

The average stress relaxation moduli of 3 animals, obtained from the experimental data of ramp and hold experiments, for different preservation periods are shown in Figure 5. The short term $\left(E_{0}\right)$ and long-term $\left(E_{\infty}\right)$ elastic modulus of bovine liver increase with an increase in preservation period.

$* * * * * * * * * * * * * * * *$ Insert Figure $5 * * * * * * * * * * * * * * * *$

In order to estimate the material coefficients of the GMS model via the optimization approach discussed above, we first obtained good initial guesses for the coefficients. This was achieved by curve fitting Prony series to the experimental data of ramp and hold experiments using Eq. 7 (Figure 6).

$* * * * * * * * * * * * * * * *$ Insert Figure $6 * * * * * * * * * * * * * * * *$ 
The residual values $\left(\mathrm{R}^{2}\right)$ suggest that the Prony series with $\mathrm{N}=3$ returns better results than $\mathrm{N}$ $=2$. Following the estimation of initial values, the optimum viscoelastic material coefficients were determined using an optimization algorithm developed in MATLAB (Table 1). In our implementation, we find a constrained minimum of the error function $F_{\min }$ (Eq. 10) of desired material coefficients starting at the initial values estimated from the relaxation data. A lower boundary was defined to prevent the optimization algorithm return negative values for the coefficients.

Table 1. The viscoelastic material coefficients of bovine liver (average of three animals) for different preservation periods.

\begin{tabular}{c|c|c|c|c|c|c|c|c|}
\hline $\begin{array}{c}\text { preservation } \\
\text { period }\end{array}$ & $\alpha_{1}$ & $\alpha_{2}$ & $\alpha_{3}$ & $\tau_{1}$ & $\tau_{2}$ & $\tau_{3}$ & $\mathbf{E}_{0}(\mathbf{k P a})$ & $\mathbf{E}_{\infty}(\mathbf{k P a})$ \\
\hline $\mathbf{1}$ & $0.09 \pm 0.01$ & $0.53 \pm 0.05$ & $0.12 \pm 0.03$ & $36.86 \pm 13.18$ & $0.44 \pm 0.32$ & $225.67 \pm 19.14$ & $18.67 \pm 0.77$ & $4.85 \pm 0.48$ \\
$\mathbf{2}$ & $0.11 \pm 0.01$ & $0.54 \pm 0.01$ & $0.11 \pm 0.00$ & $27.24 \pm 8.53$ & $0.39 \pm 0.22$ & $241.00 \pm 1.73$ & $21.81 \pm 1.60$ & $5.28 \pm 0.10$ \\
$\mathbf{4}$ & $0.12 \pm 0.03$ & $0.53 \pm 0.04$ & $0.11 \pm 0.01$ & $23.93 \pm 5.30$ & $0.31 \pm 0.05$ & $251.33 \pm 8.08$ & $23.21 \pm 1.17$ & $5.46 \pm 0.03$ \\
$\mathbf{8}$ & $0.12 \pm 0.04$ & $0.46 \pm 0.09$ & $0.18 \pm 0.05$ & $29.45 \pm 13.58$ & $0.47 \pm 0.11$ & $267.33 \pm 15.53$ & $27.08 \pm 1.23$ & $6.56 \pm 0.37$ \\
$\mathbf{1 2}$ & $0.13 \pm 0.03$ & $0.46 \pm 0.03$ & $0.19 \pm 0.04$ & $24.95 \pm 4.89$ & $0.29 \pm 0.03$ & $271.22 \pm 12.69$ & $42.54 \pm 0.74$ & $9.41 \pm 0.50$ \\
$\mathbf{1 8}$ & $0.08 \pm 0.06$ & $0.42 \pm 0.04$ & $0.25 \pm 0.02$ & $38.77 \pm 10.21$ & $0.43 \pm 0.07$ & $261.80 \pm 15.76$ & $49.47 \pm 2.15$ & $12.14 \pm 0.20$ \\
$\mathbf{2 4}$ & $0.11 \pm 0.06$ & $0.34 \pm 0.06$ & $0.26 \pm 0.02$ & $40.38 \pm 11.53$ & $0.24 \pm 0.12$ & $257.23 \pm 21.49$ & $55.63 \pm 1.30$ & $16.03 \pm 0.43$ \\
$\mathbf{3 6}$ & $0.09 \pm 0.04$ & $0.35 \pm 0.05$ & $0.27 \pm 0.01$ & $40.01 \pm 4.66$ & $0.18 \pm 0.08$ & $262.40 \pm 17.90$ & $69.91 \pm 1.02$ & $20.23 \pm 1.25$ \\
$\mathbf{4 8}$ & $0.12 \pm 0.07$ & $0.31 \pm 0.02$ & $0.28 \pm 0.03$ & $40.38 \pm 8.41$ & $0.23 \pm 0.06$ & $279.24 \pm 5.19$ & $88.00 \pm 2.65$ & $25.14 \pm 1.34$
\end{tabular}

Figure 7 compares the storage and loss moduli of bovine liver estimated from Eqs. $(8,9)$ using the initial Prony coefficients to the ones obtained from the optimization process. 
Figure 8 shows the average storage and loss moduli of 3 animals for different preservations periods. The storage modulus increases with frequency up to the resonance frequency first and then stays almost constant after that (see Figure 8a). The loss modulus also increases with frequency, reaching to a peak value at resonance frequency (maximum energy dissipation occurs at the resonance), but then decreases to zero as the frequency is further increased (see Figure $8 \mathrm{~b}$ ). The storage and loss moduli of bovine liver estimated in this study for $\mathrm{T}=1-4 \mathrm{hrs}$ (5-20 $\mathrm{kPa}$ for $\mathrm{E}_{\mathrm{S}}(\omega)$ and 1-5 $\mathrm{kPa}$ for $\left.\mathrm{E}_{\mathrm{L}}(\omega)\right)$ are comparable to the ones reported for bovine liver (1-6 kPa for $\mathrm{E}_{\mathrm{S}}(\omega)$ and $\left.\mathrm{E}_{\mathrm{L}}(\omega)<1 \mathrm{kPa}\right)$ in Liu and Bilston (2000), for fresh veal liver (10$40 \mathrm{kPa}$ ) in Zhang et al. (2007), for canine liver (1-10 kPa) in Kiss et al. (2004) and the magnitude of complex shear modulus values reported for porcine liver (5-50 kPa) in Valtorta and Mazza (2005). Since the storage and loss moduli are related to the energy storage and dissipation capacities of the tissue respectively, the results of the impact experiments are well aligned with that of the ramp and hold experiments. For example, the storage modulus of the bovine liver tested at $\mathrm{T}=48$ hours is more than 4 times higher than that of the one tested at $\mathrm{T}$ $=1$ hours (Figure $8 \mathrm{a}$ ). This is due to the fact that the former is more than 4 times stiffer than the latter (Figure 9a). The long-term (i.e. steady-state) elastic modulus values of bovine liver estimated in this study for $\mathrm{T}=1-4 \mathrm{hrs}(\sim 5 \mathrm{kPa})$ is highly compatible with the value obtained for bovine liver $($ shear modulus $=0.6 \mathrm{kPa})$ in Liu and Bilston $(2000)$, for pig liver $(\sim 10 \mathrm{kPa})$ in Ottensmeyer (2001), Kruse et al. (2000), Kim et al. (2006), Samur et al. (2007) and for human liver ( $20 \mathrm{kPa})$ in Nava et al. (2008). It also appears that the relation between the longterm elastic modulus and the preservation time is linear. For the same amount of compression, a stiffer material stores more energy than the softer one. The similar argument can be made for the loss modulus (Figure $8 \mathrm{~b}$ ). The increase in the loss modulus of bovine liver as a 
function of preservation period is an indication for an increase in energy dissipation, which is caused by the damping in the material. As the damping increases, the time constant of the liver increases and the liver responds more slowly to the external loading.

***************** Insert Figure $8 * * * * * * * * * * * * * * * *$

****************** Insert Figure $9 * * * * * * * * * * * * * * * *$

In order to get a better idea about the relaxation time constants, we estimate the settling time of the relaxation curves for different preservation periods using a percent relative error, $R E$, defined as

$R E=100\left(E_{R}(t)-E_{\infty}\right) / E_{\infty}$

In the calculation of settling times, the threshold for the relative error was taken as $R E_{\text {threshold }}$ $=5 \%$. The relaxation response of the liver tissue slows down as it spends more time in the preservation cycle (Figure 9b). However, it appears that the settling time does not follow a linear relation with the preservation period. For example, the relaxation response of the liver sample tested at $\mathrm{T}=48$ hours is approximately 2 times slower (more viscous) than that of the one tested at $\mathrm{T}=1$ hours. These results support the earlier findings suggesting that excised liver tissue becomes stiffer (Kerdok et al., 2006 and Rosen et al., 2008) and more viscous (Kerdok et al., 2006) in time. 


\section{Conclusion}

In liver transplantation, the donor and the recipient are typically in different locations, which bring up the problem of the preservation. Unfortunately, there is no standard among the physicians on how long the preservation period must be. In the simple hypothermic preservation approach, first, the harvested liver is flushed with an appropriate chemical solution, and then immersed into a plastic bag containing the same solution; finally the bag is covered with ice. The chemical solutions suggested in the literature for preserving a harvested liver differ slightly in components, but they all aim to prevent swelling of liver cells and delay their destruction, which is inevitable. While the effect of preservation period on the cell structure and functionality of animal and human livers have been investigated extensively, the same effect on the gross material properties of liver tissue has been mostly neglected.

In this article, we investigated the effect of preservation period on the dynamic (both time and frequency-dependent) material properties of bovine liver with implications for liver transplantation. In our study, the time-dependent relaxation moduli of bovine liver for different preservation periods were measured using a compression set-up developed in our laboratory. On the other hand, the frequency-dependent material characteristics of the same liver samples were measured for different preservation periods using a commercial impact hammer. Frequency-dependent viscoelastic material properties of soft tissues are typically characterized by dynamic loading test, which can be induced either by a rheometer or a mechanical vibrator. We showed that an alternative approach for the same purpose is the impulse loading via an impact hammer. Compared to the dynamic loading test, the measurement time in impact test is much shorter. The technique simply involves the use of a hand-held hammer to apply a light impact force on a pre-load mass placed on the top surface of a specimen. The hammer incorporates a sensor that produces a signal proportional to the 
force of impact. This enables precise measurement of the excitation force. Different impact tip materials allow tailoring of the frequency content of the impact force. For low frequency measurements as in our case, a soft rubber tip concentrates the excitation energy in a narrow frequency range.

In order to obtain the optimum viscoelastic material coefficients of bovine liver, we fit the data collected from the both experiments (relaxation and impact) to a Generalized Maxwell Solid (GMS) model. In earlier studies focusing on viscoelastic material properties of soft tissues have typically relied on the experimental data collected from one type of experiment only. Either relaxation or dynamic loading experiments are performed to model time- or frequency-dependent material properties of the soft tissues being tested, respectively. However, due to the nature of these experiments, the information that can be extracted from each one is different though a conversion from time to frequency domain or vice versa is possible through Laplace transformations. We showed that a better fit to the proposed viscoelastic tissue model can be achieved if the results of both experiments are taken into account in the analysis.

Using the material coefficients estimated through the viscoelastic model, we investigated the effect of preservation period on the material properties of bovine liver. Our analysis showed that the liver tissue becomes stiffer and more viscous as it spends more time in the preservation cycle. It is important to note that these results must be evaluated with caution since the proposed approach utilizes a linear viscoelastic model to investigate the effect of preservation time on the tissue response, and fails to consider the material nonlinearities and rate-dependent viscoelastic effects, which are also important in material characterization. 


\section{Acknowledgements}

The Scientific and Technological Research Council of Turkey, TUBITAK, partially supported this work under contract MAG-104M283.

\section{References}

1. Carter, F.J., Frank, T.G., Davies, P.J., McLean, D., Cuschieri, A., 2001. Measurement and modeling of the compliance of human and porcine organs. Medical Image Analysis, Vol. 5, pp. 231-236.

2. Liu, Z., Bilston, L., 2000, On the viscoelastic character of liver tissue: experiments and modeling of the linear behavior, Biorheology, Vol. 37, No. 3, pp. 191-201.

3. Tay, B. K., Kim, J., and Srinivasan, M. A., 2006, "In vivo mechanical behavior of intra-abdominal organs,” IEEE Transaction on Biomedical Engineering, Vol. 53, No. 11, pp. 2129-2138.

4. Kiss, M. Z., Varghese, T., Hall, T. J., 2004, Viscoelastic characterization of in vitro canine tissue, Physics in Medicine and Biology, Vol. 49, No. 18, pp. 4207-4218.

5. Samur, E., Sedef, M., Basdogan, C., Avtan, L., Duzgun, O., 2007, A robotic indenter for minimally invasive measurement and characterization of soft tissue behavior, Medical Image Analysis, Vol. 11, No.4, pp. 361-373.

6. Roan, E., Vemaganti, K., 2007, The non-linear material properties of liver tissue determined from no-slip uniaxial compression experiments, ASME Journal of Biomechanical Engineering, Vol. 129, pp. 450-456

7. Kerdok, A. E., Ottensmeyer, M. P., and Howe, R. D., 2006, Effects of perfusion on the viscoelastic characteristics of liver, J. Biomechanics, Vol. 39, No. 12, pp. 2221-2231. 
8. Rosen J, Brown JD, De S, Sinanan M, Hannaford B., 2008, Biomechanical properties of abdominal organs in vivo and postmortem under compression loads, ASME Journal of Biomechanical Engineering, Vol. 130, No. 2, pp. 021020-1-021020-16.

9. Mazza, E., Nava, A., Hahnloser, D., Jochum, W., Bajka, M., 2007, The mechanical response of human liver and its relation to histology: An in vivo study, Medical Image Analysis, Vol. 11, pp. 663-672.

10. Valtorta D., Mazza E., 2005, Dynamic measurement of soft tissue viscoelastic properties with a torsional resonator device, Medical Image Analysis, Vol. 9, pp. 481490.

11. Lin, T.R., Farag, N.H., Pan, J., 2005, Evaluation of frequency dependent rubber mount stiffness and damping by impact test, Applied Acoustics, Vol. 66, No. 7, pp. 829-844.

12. Nashif, A.D., Jones, D., Henderson, J.P., 1985, Vibration damping, Wiley.

13. Nava, A., Mazza, E., Furrer, M., Villiger, P., Reinhart, W.H., 2008, In vivo mechanical characterization of human liver, Medical Image Analysis, Vol. 12, pp. 203-216.

14. Saraf, H., Ramesh, K.T., Lennon, A.M., Merkle, A.C., Roberts, J.C., 2007, Mechanical properties of soft human tissues under dynamic loading, Journal of Biomechanics, Vol. 40, No. 9, pp. 1960-1967.

15. Zhang, M., Castaneda, B., Wu, Z., Nigwekar, P., Joseph, J., Rubens, D.J., Parker, K.J., 2007, Congruence of imaging estimator and mechanical measurements of viscoelastic properties of soft tissues. Journal of Ultrasound in Medicine and Biology, Vol. 33, No. 10, pp. 1617-1631.

16. Kruse, S.A., Smith J.A., Lawrence, A.J., Dresner, M.A., Manduca A., Greenleaf, J.F., Ehman, R.L., 2000, Tissue characterization using magnetic resonance elastography: Preliminary results, Physics in Medicine and Biology, Vol. 45, No. 6, pp. 1579-1590. 


\section{List of Table Captions:}

Table 1. The viscoelastic material coefficients of bovine liver (average of three animals) for different preservation periods. 


\section{List of Figure Captions:}

Figure 1. The set-up for conducting impact experiments to determine the storage and loss moduli of bovine liver.

Figure 2. The set-up for conducting ramp and hold experiments to characterize the stress relaxation modulus of bovine liver.

Figure 3. The flow-chart of the optimization process for estimating the viscoelastic material coefficients of soft organ tissues.

Figure 4. The storage (a) and loss (b) moduli of bovine liver measured at 1, 2, 4, 8, 12, 18, 24, 36 and 48 hours after harvesting (raw experimental data for one animal).

Figure 5. The stress relaxation modulus of bovine liver measured at 1, 2, 4, 8, 12, 18, 24, 36 and 48 hours after harvesting (filtered experimental data for 3 animals).

Figure 6. The stress-relaxation response of bovine liver is estimated via curve fitting a Prony series with $\mathrm{N}=2$ (blue solid) and $\mathrm{N}=3$ (red solid) to the experimental data (dashed) collected 1 hour after harvesting (only the first 20 seconds of the data is displayed for comparison).

Figure 7. The storage (a), and loss (b) moduli estimated through the stress relaxation response (solid blue) are compared to that of the optimization process (the solid red curves represent the solution obtained locally) for the experimental data collected one hour after harvesting (dashed).

Figure 8. The storage (a) and loss (b) moduli of bovine liver estimated through the optimization process for the preservation periods of $1,2,4,8,12,18,24,36$ and 48 hours.

Figure 9. a) The variation in the long-term (steady state) elastic modulus of bovine liver as a function of preservation period for 3 different animals $\left(\mathrm{E}_{\infty}=0.45^{*} \mathrm{~T}+4.1, \mathrm{R}^{2}=0.98\right)$. b) The variation in the settling time of bovine liver as a function of preservation period (Settling Time $\left.=80.3 * \log (\mathrm{T})+333.3, \mathrm{R}^{2}=0.90\right)$ 


\section{List of Tables:}

\begin{tabular}{c|c|c|c|c|c|c|c|c|}
\hline $\begin{array}{c}\text { preservation } \\
\text { period }\end{array}$ & $\alpha_{1}$ & $\alpha_{2}$ & $\alpha_{3}$ & $\tau_{1}$ & $\tau_{2}$ & $\tau_{3}$ & $\mathbf{E}_{0}(\mathbf{k P a})$ & $\mathbf{E}_{\infty}(\mathbf{k P a})$ \\
\hline $\mathbf{1}$ & $0.09 \pm 0.01$ & $0.53 \pm 0.05$ & $0.12 \pm 0.03$ & $36.86 \pm 13.18$ & $0.44 \pm 0.32$ & $225.67 \pm 19.14$ & $18.67 \pm 0.77$ & $4.85 \pm 0.48$ \\
$\mathbf{2}$ & $0.11 \pm 0.01$ & $0.54 \pm 0.01$ & $0.11 \pm 0.00$ & $27.24 \pm 8.53$ & $0.39 \pm 0.22$ & $241.00 \pm 1.73$ & $21.81 \pm 1.60$ & $5.28 \pm 0.10$ \\
$\mathbf{4}$ & $0.12 \pm 0.03$ & $0.53 \pm 0.04$ & $0.11 \pm 0.01$ & $23.93 \pm 5.30$ & $0.31 \pm 0.05$ & $251.33 \pm 8.08$ & $23.21 \pm 1.17$ & $5.46 \pm 0.03$ \\
$\mathbf{8}$ & $0.12 \pm 0.04$ & $0.46 \pm 0.09$ & $0.18 \pm 0.05$ & $29.45 \pm 13.58$ & $0.47 \pm 0.11$ & $267.33 \pm 15.53$ & $27.08 \pm 1.23$ & $6.56 \pm 0.37$ \\
$\mathbf{1 2}$ & $0.13 \pm 0.03$ & $0.46 \pm 0.03$ & $0.19 \pm 0.04$ & $24.95 \pm 4.89$ & $0.29 \pm 0.03$ & $271.22 \pm 12.69$ & $42.54 \pm 0.74$ & $9.41 \pm 0.50$ \\
$\mathbf{1 8}$ & $0.08 \pm 0.06$ & $0.42 \pm 0.04$ & $0.25 \pm 0.02$ & $38.77 \pm 10.21$ & $0.43 \pm 0.07$ & $261.80 \pm 15.76$ & $49.47 \pm 2.15$ & $12.14 \pm 0.20$ \\
$\mathbf{2 4}$ & $0.11 \pm 0.06$ & $0.34 \pm 0.06$ & $0.26 \pm 0.02$ & $40.38 \pm 11.53$ & $0.24 \pm 0.12$ & $257.23 \pm 21.49$ & $55.63 \pm 1.30$ & $16.03 \pm 0.43$ \\
$\mathbf{3 6}$ & $0.09 \pm 0.04$ & $0.35 \pm 0.05$ & $0.27 \pm 0.01$ & $40.01 \pm 4.66$ & $0.18 \pm 0.08$ & $262.40 \pm 17.90$ & $69.91 \pm 1.02$ & $20.23 \pm 1.25$ \\
$\mathbf{4 8}$ & $0.12 \pm 0.07$ & $0.31 \pm 0.02$ & $0.28 \pm 0.03$ & $40.38 \pm 8.41$ & $0.23 \pm 0.06$ & $279.24 \pm 5.19$ & $88.00 \pm 2.65$ & $25.14 \pm 1.34$
\end{tabular}


List of Figures (color online only):

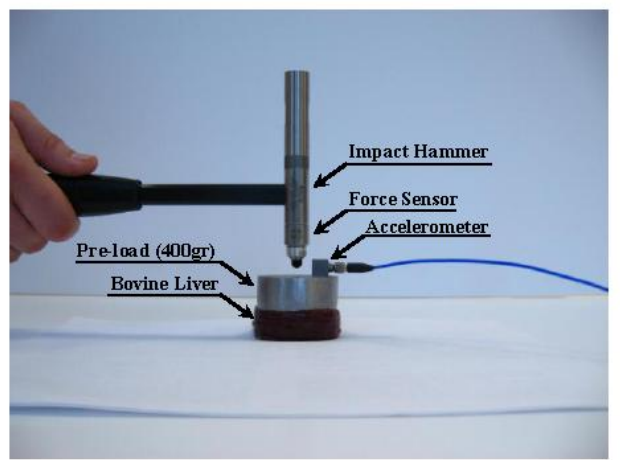




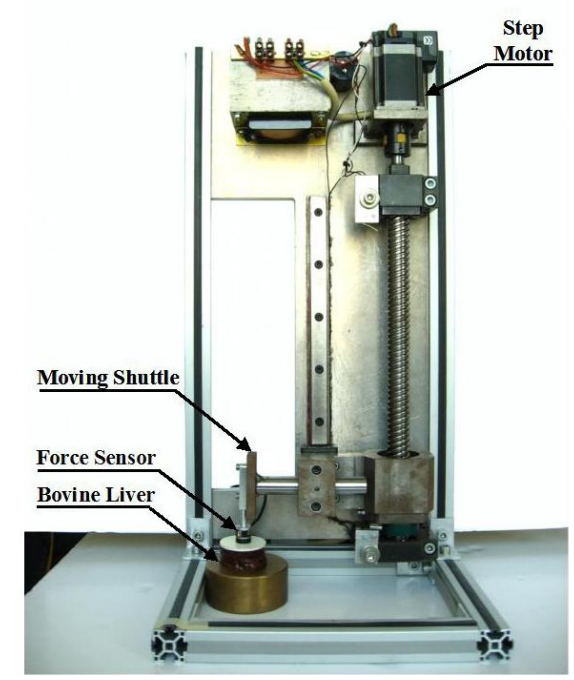




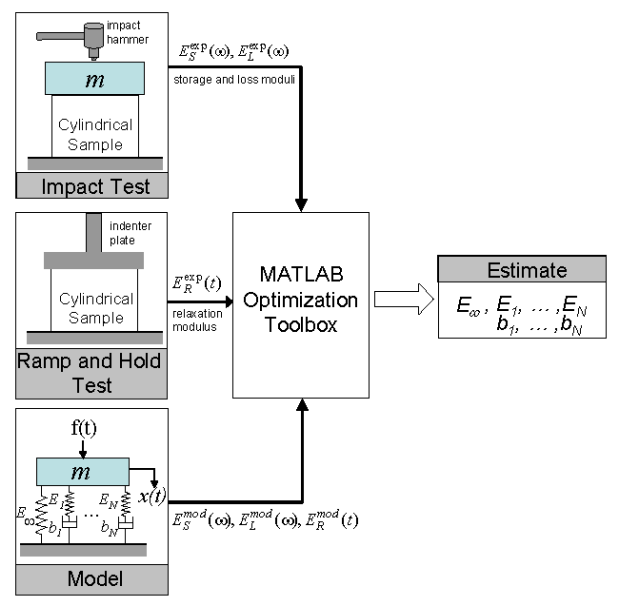




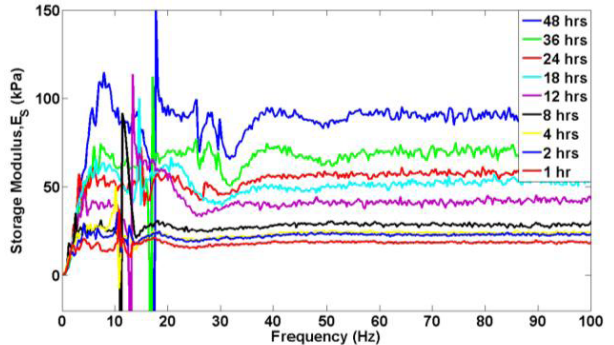

(a)

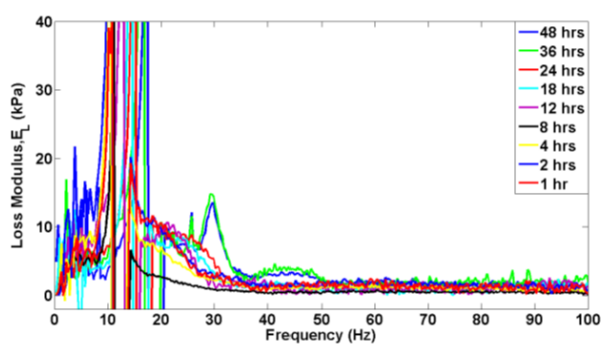

(b) 


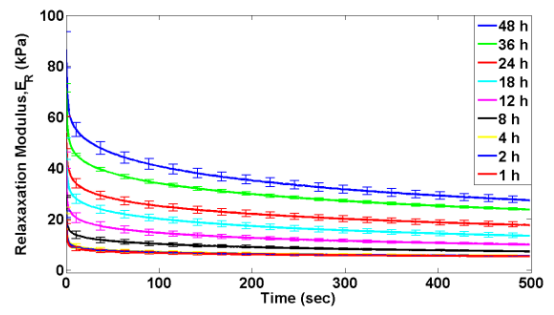




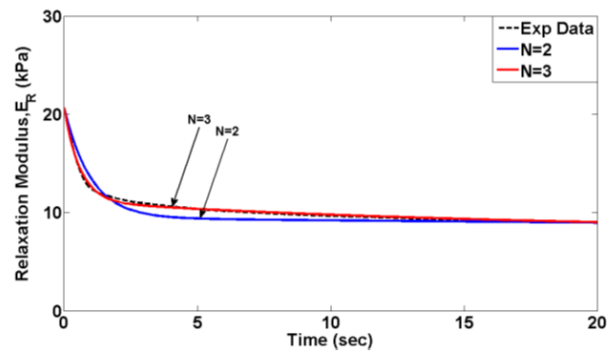




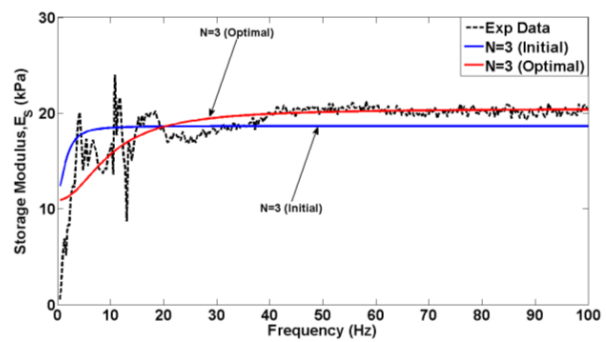

(a)

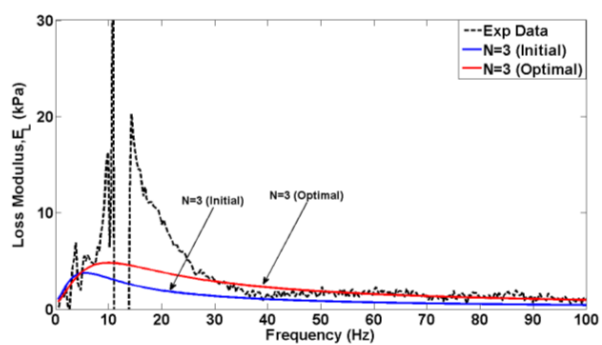

(b) 


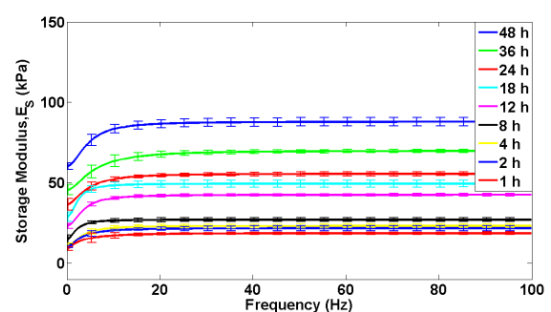

(a)

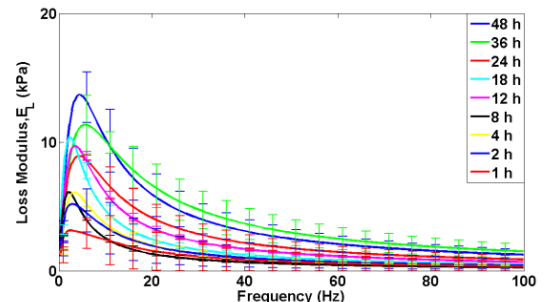

(b) 


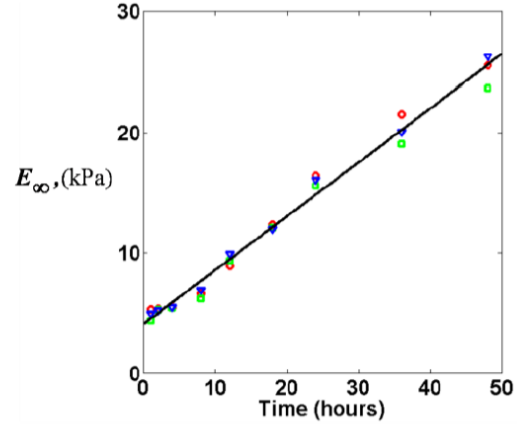

(a)

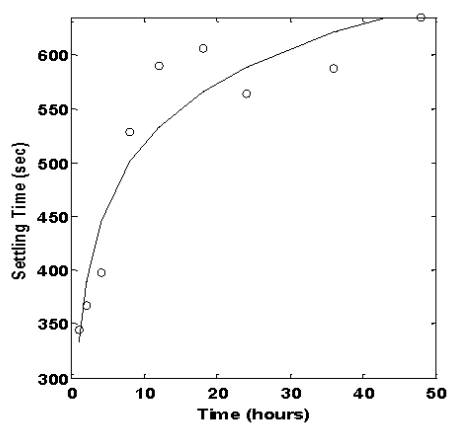

(b) 